\title{
Measurement of Wear in Radial Journal Bearings
}

\author{
D.J. Ligterink and A.W.J. de Gee \\ Tribology Section, Faculty of Mechanical Engineering, \\ University of Twente, Enschede \\ The Netherlands
}

Abstract

In this article, the measurement of wear in radial journal bearings is discussed, where a distinction is made between stationary and non-stationary contact conditions. Starting with Holm/Archard's wear law, equations are derived for the calculation of the specific wear rate $\mathrm{k}$ of the bearing material as a function of the wear depth $\mathrm{d}$, measured after an experiment or a period of use in practice. It is also possible to calculate a value for the maximum allowable apparent pressure $\mathrm{p}_{a}$ at given values of $\mathrm{k}$, required lifetime and maximum allowable wear depth $\mathrm{d}$, or a value of $\mathrm{d}$ at a given value of $\mathrm{p}_{a}$. In order to facilitate the use of the equations mentioned above, non-dimensional diagrams are presented. Two examples are given to explain the use of these diagrams.

Keywords wear measurement, radial, journal, bearing, stationary contact, nonstationary

Nomenclature

$A$ area of cross-section of worn part

$b$ width of wear scar

$d$ wear depth

$F$ radial force

$k$ specific wear rate

$L$ bearing length

$n$ total number of revolutions

$p_{\mathrm{a}}$ apparent pressure

$r$ radius of journal

$s$ sliding distance

$V$ wear volume

$\Delta r$ radial clearance

$\sigma \Delta r / r$

$\delta d / r$

INTRODUCTION Under conditions of full-film lubrication, the thickness of the lubricant film is supposed to be large enough to separate the 
roughness peaks on the surfaces of journal and bushing completely. If full-film lubrication of a bearing cannot be guaranteed during its entire lifetime, the wear rate (defined as the gradual loss of material of the bearing bush, caused by mechanical contact with the journal, i.e. the journal is assumed not to suffer from wear, and erosion and surface fatigue do not occur) of the bearing material is an important quality criterion that should be taken into account by designers and users. This is particularly true if the bearing is designed to function dry, in which case a polymerbased bearing material will often be used, ${ }^{1}$ or if 'pure' boundary lubrication conditions apply. In the latter case, separation of the (usually metallic) roughness peaks is achieved by means of a thin layer, strongly bonded to the surfaces. ${ }^{2}$

It is not usually possible to predict the wear rate of a newlydeveloped bearing material on the basis of primary data on the journal and bushing materials such as, for instance, hardness, Young's modulus, or heat conductivity. This is primarily due to the 'system character' of wear which, among other things, implies that the wear rate of a bearing material depends on its interaction with the material of the journal and with the lubricant. ${ }^{3}$ As a consequence, one has to rely on the results of experiments. In such experiments, one often uses a small stationary specimen, made of the bearing material to be characterised, in contact with a rotating (usually steel) shaft. Unfortunately, the use of small specimens may lead to a serious discrepancy between laboratory test results, and wear behaviour in practice. Important reasons for such discrepancy may be differences in:

- trapping of wear particles between the contacting surfaces

- friction-induced contact temperature*

- supply of lubricant to the contact zone

- entry of oxygen into the contact zone

- friction-induced vibrations.

Because of this, it is recommended to use radial journal bearings as test pieces, rather than small specimens.

If the sizes of the test bearings differ significantly from those in practice (which will frequently be the case), the transferability of the test results from the laboratory to practice is enhanced by performing the tests under the apparent pressure $p_{\mathrm{a}}$ (defined as

* The occurrence of friction between bushing and journal causes 'frictional heat', which may lead to high contact temperatures (sometimes of the order of $500^{\circ} \mathrm{C}$ ). Obviously, the equilibrium contact temperature is also a function of the potential for heat transport in the bearing. 
Figure 1 Schematic presentation of radial journal bearings operating under conditions of nonstationary contact ( $a$ and $b$ ) and stationary contact $(c)$

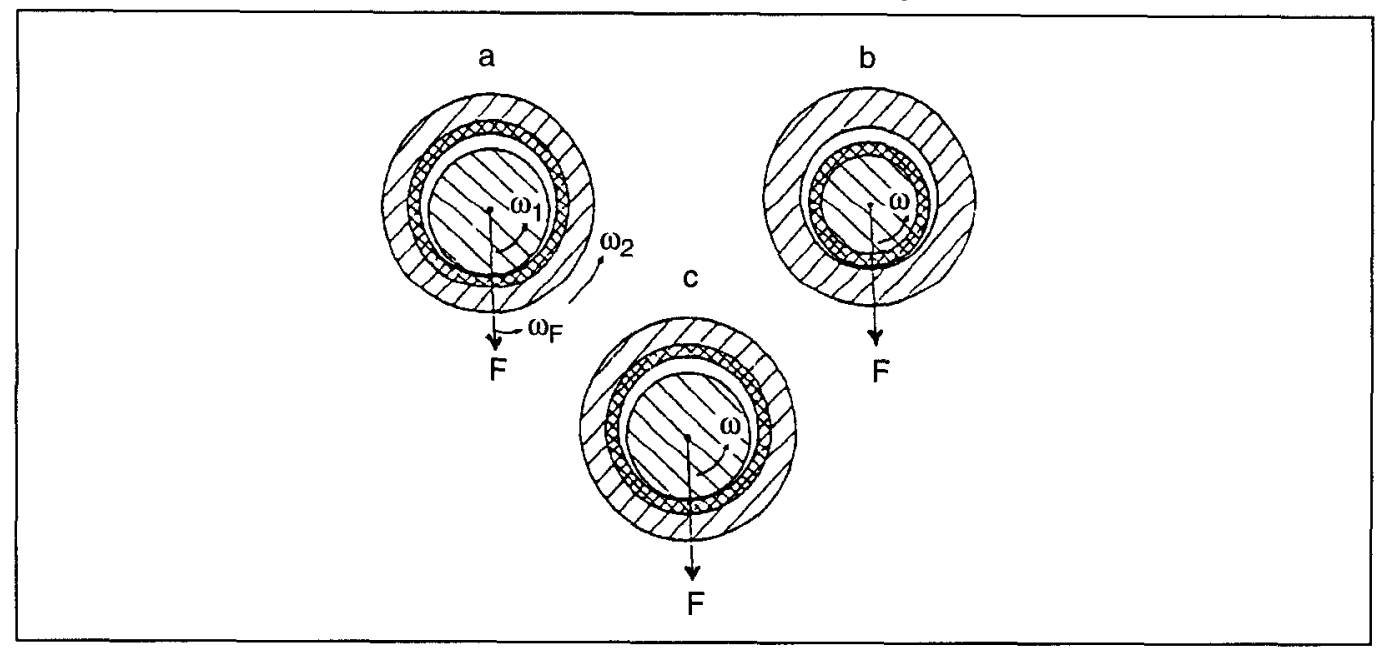

the force $F$ on the bearing, divided by the product of bearing diameter $D$ and bearing length $L$ ) that is expected to occur in practice. If lubrication is to be supplied, another prerequisite for successful simulative testing is that the lubricant in the tests should be the same as that in practice. ${ }^{3}$

In line with the requirements of ISO 7148 , a state of 'pure' boundary lubrication should be achieved during the tests, entirely avoiding a state of mixed lubrication. In tests performed in the presence of a lubricant, this usually implies application of a low angular velocity of the journal, resulting in a linear velocity of the order of $0.01 \mathrm{~m} / \mathrm{s}$ (see reference 4 for an example of the application of ISO 7148). Obviously, such a velocity restriction does not apply to dry running bearings.

Finally if, in testing, a radial journal bearing is used, the bushing should be aligned carefully to obtain a wear track of constant width. It should preferably be self-aligning with respect to the mechanism that transmits the force on the bearing.

Calculation of the specific wear rate $k$

The starting point for the calculation of $k$ is Holm/Archard's law: ${ }^{5,6}$

$k=\frac{V}{F S}$

in which

$V=$ volume of material removed as a result of wear $\left(\mathrm{m}^{3}\right)$ 
$F=$ radial force on the bearing $(\mathrm{N})$

$s=$ total sliding distance $(\mathrm{m})$

(Eq. 1 can also be rewritten defining a wear modulus which replaces $k$. The unit of this wear modulus is $\mathrm{N} / \mathrm{m}^{27,8}$ ) According to Archard, $k$ is constant as long as the wear mechanism does not change.

Prior to applying eq. 1 to a radial journal bearing, a distinction should be made between bearings operating under conditions of non-stationary contact, and those operating under conditions of stationary contact. ${ }^{9}$ Under non-stationary contact conditions, the location of the contact zone between bushing and journal rotates with respect to the surface covered with bearing material. This will be the case if the bushing or the load rotates (Figure 1a), or if the bearing material to be characterised is present on the journal, rather than on the inner side of the bushing (Figure 1b). The latter case may occur if the bearing surface material is applied by means e.g. of metal spraying.

Under stationary contact conditions, the location of the contact zone between bushing and journal does not rotate with respect to the surface covered with the bearing material.

This will be the case if neither the bushing nor the load rotates and the bearing material is present on the inner surface of the bushing (Figure 1c). It can be noted that a dynamically loaded bearing, in which the magnitude of the load varies, but in which the direction of the load is constant, operates under conditions of stationary contact.

Non-stationary contact Under conditions of non-stationary contact, wear of the bearing material results in removal of material, uniformly distributed along the inner surface of the bushing or the outer surface of the journal. The latter case is depicted in Figure 2, in which the material removed by wear is identified by hatching. This removal results in a continuous increase in radial clearance $\Delta r$ and, usually, a gradual deterioration of the condition of lubrication. This is because, in designing a hydrodynamically lubricated radial journal bearing, the value of the radial clearance is usually optimised with respect to the lubricant film thickness, friction or stiffness. ${ }^{10 t}$

* In radial journal bearings, operating under conditions of mechanical contact between bushing and journal, the following wear mechanisms may occur; thermal wear (wear due to local melting), corrosive wear, deformation wear, wear by delamination, adhesive wear, wear by micro-cutting and wear by surface fatigue at macro- or micro scale. A change in mechanism may be the result of a change in test conditions, notably in sliding velocity; see also the footnote on page 52.

$\dagger$ Note that a significant decrease in surface roughness as a result of contact between bushing and journal may counteract the undesirable effect of wear. 
Figure 2 Uniform removal by wear of bearing material present on a journal. The bearing is operating under conditions of non-stationary contact (see Figure 1b)

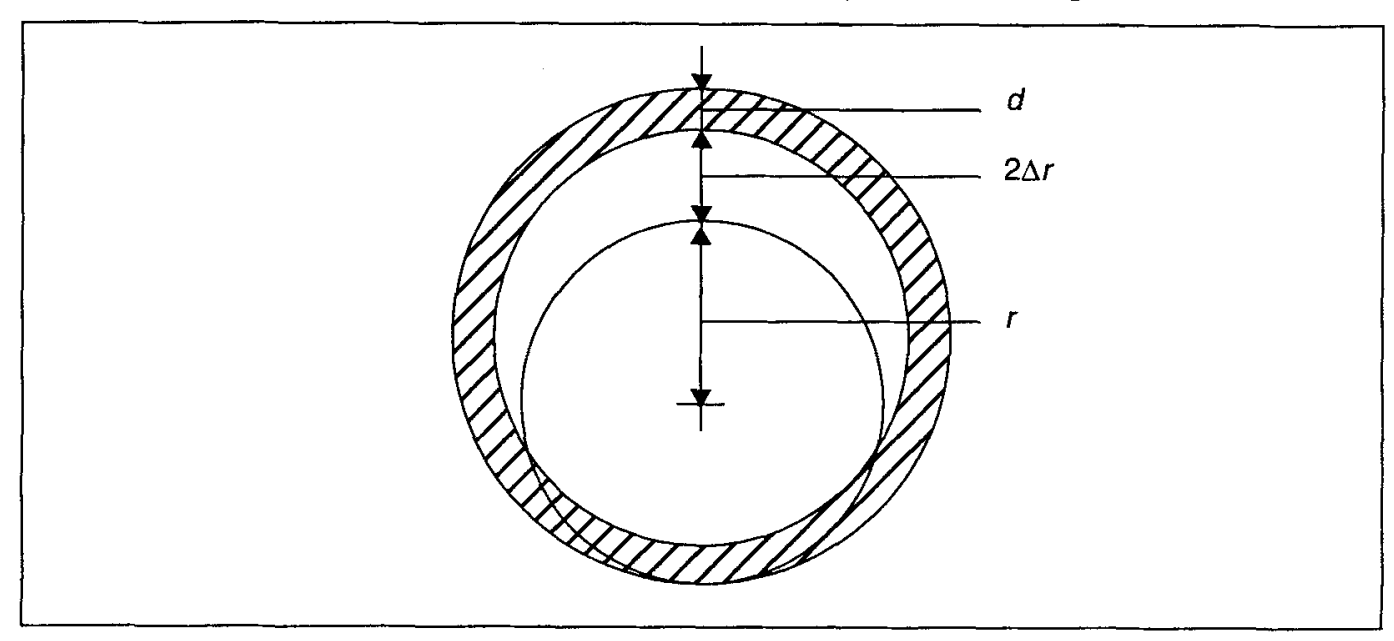

In laboratory testing, a non-stationary contact condition will occur if the bearing material to be characterised is present on the journal (Figure 1b). If $r$ is the radius of the journal and $d$ the displacement of the journal as a result of wear, the area $A$ of the crosssection of the worn part is given by:

$$
A=\pi\left[2 r d+d^{2}\right]
$$

Substituting eq. 2 in eq. 1 with $V=A L, F=2 r p_{\mathrm{a}} L(L=$ bearing length), $s=2 \pi r n$ ( $n=$ total number of revolutions of the journal), $\sigma=\Delta r / r$ and $\delta=d / r$, results in:

$k=\frac{2 \delta+\delta^{2}}{4 p_{\mathrm{a}} n}$

Inserting realistic values for $\sigma$ and $\delta$, e.g. $\sigma=10^{-3}$ and $\delta=10^{-3}$, shows that the second order term in eq. 3 can be neglected. This leads to:

$k=\frac{\delta}{2 p_{\mathrm{a}} n}$

In this case, $\delta(d)$ can be found by measuring the radius of the journal before and after the test, subtracting the data thus found.

Eq. 4 can also be used the other way round. For a given value of $k$, a maximum allowable value of $d$ (important with 
Figure 3 Removal of material by wear in a bushing. The bearing is operating under conditions of stationary contact (see Figure 1c)

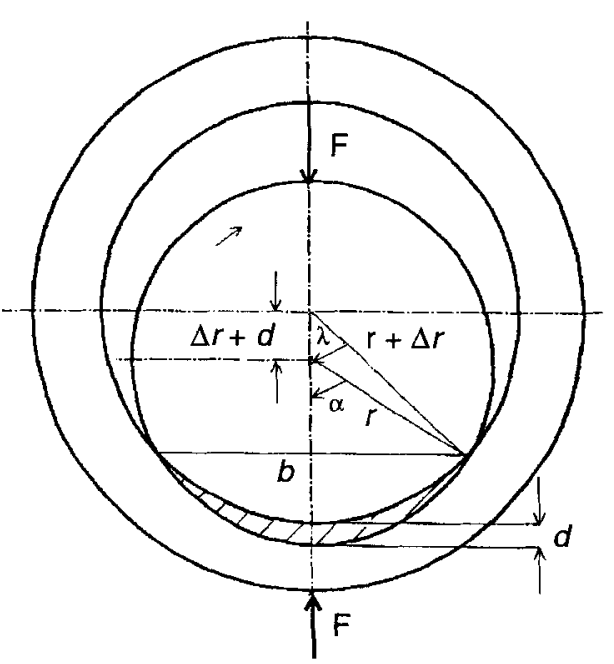

respect to positioning of the shaft) and a required lifetime (value of $n$ ), the value of $p_{\mathrm{a}}$ can be calculated. In this case, $p_{\mathrm{a}}$ represents the maximum allowable value of the apparent contact pressure. For given values of $r$ and $F$ and with $L=F / 2 r p_{a}$, this yields the required value of the bearing length $L$.

Stationary contact Figure 3 depicts the situation under conditions of stationary contact. Here also, the hatching identifies the bearing material removed as a result of wear. The journal has penetrated the bushing over a depth $d$.

If the surface roughness of the bearing material does not increase as a result of wear, a wear pattern as shown in Figure 3 will usually improve the lubrication condition. ${ }^{9}$ However, at a given wear volume $V$, the displacement of shaft as a result of wear will be larger than in the case of non-stationary contact.

According to reference 11, the area $A$ of the cross-section of the wear scar can now be found (see also Figure 3 ) from:

$A=\frac{r^{2}}{2}[2 \alpha-\sin 2 \alpha]-\frac{(r+\Delta r)^{2}}{2}[2 \lambda-\sin 2 \lambda]$

For $\alpha$ and $\lambda$ one easily finds:

$$
\alpha=\arccos \frac{2 \sigma-2 \sigma \delta-\delta^{2}}{2(\sigma+\delta)}
$$


and

$\lambda=\arccos \frac{2 \sigma+2 \sigma^{2}+2 \sigma \delta+\delta^{2}}{2(1+\sigma)(\sigma+\delta)}$

which results in the following equation for $k$ :

$k=\frac{2 \alpha-\sin 2 \alpha-(1+\sigma)^{2}(2 \lambda-\sin 2 \lambda)}{8 \pi p_{\mathrm{a}} n}$

Analysis shows that, in this case, second order terms cannot be neglected. With eq. $8, k$ can be calculated from data on the bearing geometry $(r, \Delta r, L), F, n$ and $d$.

In this case, the value of $d$ can be determined in two different ways:

- directly, i.e. by measuring the displacement of the bushing in the direction of the force, during performance of the test. This can, for instance, be done with a differential transformer technique, as described in reference 12

- by comparing the surface profile of a worn bushing with that of the same bushing before the test. This can be done with a profile (roughness) measuring instrument, adapted for the measurement of hollow surfaces over a large measuring length.

The width $b$ of the wear scar also forms a measure for the wear depth $d$, because $b$ and $\alpha$ (eq. 6) are related according to:

$b=2 r \sin \alpha$

However, for the usually very small values of $\sigma$ (order $10^{-3}$ ), very small differences in $b$ lead to considerable differences in $\delta$, which makes this method extremely unreliable.

Once again, it is possible to use eq. 8 for calculating $p_{a}$ for given values of $k, r, \Delta r, d$ and $n$ or, for a given value of $F$, to calculate $L$.

Because of the rather complicated character of eqs. 6, 7 and 8 , it is not possible to make a quick estimate of the effect of a variation in one of the parameters on the values of the others.

Thus, it is convenient to use a graphic presentation of eq. 8 in which the (dimensionless) product of $p_{a}, k$ and $n$ is plotted as a

* From this information the value of A also can be found by (graphic) determination of the area of the surface, enclosed by the two profiles. 
Figure 4 Value of the product $p_{\mathrm{a}} k n$ as a function of the relative wear depth $\delta$ for four values of the relative radial clearance $\sigma$, with $2.10^{-4} \leq \delta \leq 20.10^{-4}$

a: $\sigma=2 \cdot 10^{-4}, b: \sigma=5 \cdot 10^{-4}, c: \sigma=10.10^{-4}, d: \sigma=20.10^{-4}$

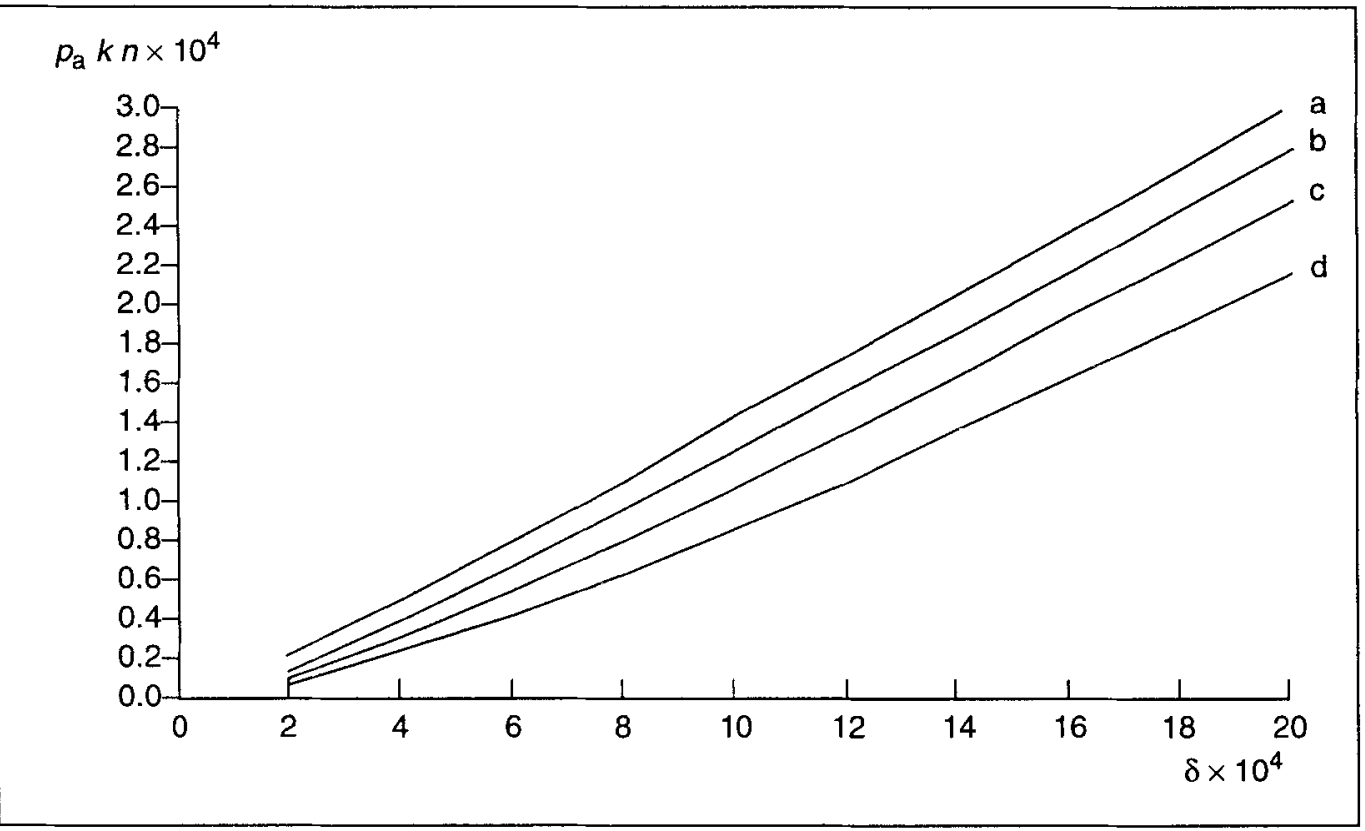

function of $\delta$. Figures 4 and 5 show such presentations for, respectively, $2.10^{-4} \leq \delta \leq 20.10^{-4}$ and $2.10^{-4} \leq \delta \leq 100.10^{-4}$. Both diagrams show four curves, corresponding with different, realistic, values of $\sigma .^{10}$

Use of Figures 4 The following samples illustrate the use of Figures 4 and 5.

Calculation of $\mathrm{k}$ on the basis of a measured value of $\mathrm{d}$ Under conditions of boundary lubrication, a radial journal bearing with $r$ $=0.02 \mathrm{~m}$ is loaded with an apparent pressure $p_{\mathrm{a}}$ of $10^{7} \mathrm{~N} / \mathrm{m}^{2}$. The sliding speed $v$ in the interface is $0.01 \mathrm{~m} / \mathrm{s}$, the radial clearance $\Delta r$ is $2.10^{-5} \mathrm{~m}$, i.e. $\sigma=10.10^{-4}$. After $10^{5}$ revolutions of the journal (i.e. $n=10^{5}$ ), the wear depth $d$ is equal to $1.10^{-4} \mathrm{~m}$, i.e. $\delta^{\prime} \approx 50 \cdot 10^{-4}$. For the given values of $\sigma$ and $\delta$, Figure 5 yields a value for $p_{\mathrm{a}} k n$ of $7.10^{-4}$. With $n=10^{5}$ and $p_{\mathrm{a}}=10^{7} \mathrm{~N} / \mathrm{m}^{2}$, this results in a $k$ value of $7.10^{-16} \mathrm{~m}^{3} / \mathrm{Nm}$.

* Eq. 8 does not contain the sliding speed $v$. Nevertheless, $k$ may well depend on $v$, because for instance the friction-induced contact temperature increases with increasing $u$. This is particularly important if uniubricated polymers are characterised at $v>0.01 \mathrm{~m} / \mathrm{s}^{12,13}$ 
Figure 5 Value of the product $p_{\mathrm{a}} k n$ as a function of the relative wear depth $\delta$ for four values of the relative radial clearance $\sigma$, with $20.10^{-4} \leq \delta \leq 100.10^{-4}$

a: $\sigma=2.10^{-4}, b: \sigma=5 \cdot 10^{-4}, c: \sigma=10.10^{-4}, \mathrm{~d}: \sigma=20.10^{-4}$

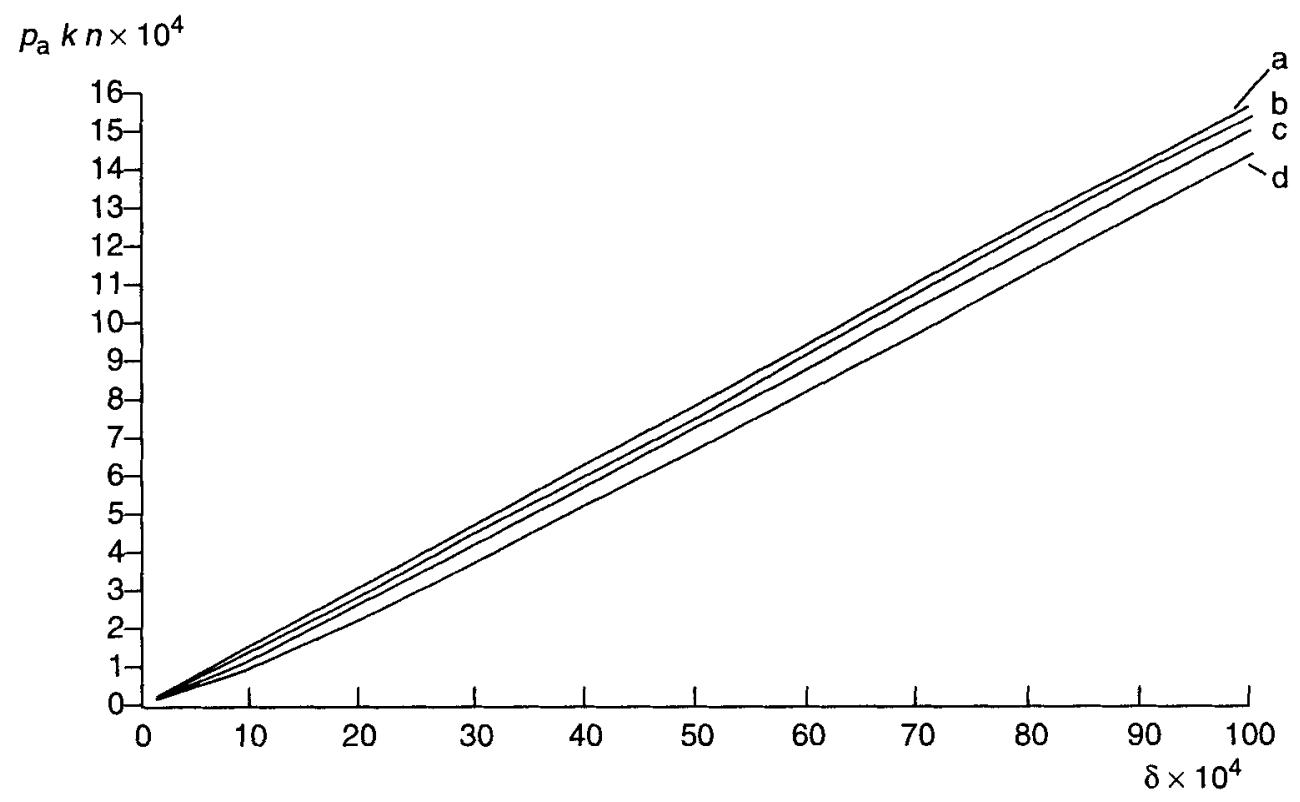

Characterisation of a journal bearing on the basis of a $\mathrm{d}$ value, estimated with Figure 4 Because of positioning requirements, the wear depth $d$ in a bearing should not exceed $4.10^{-6} \mathrm{~m}$ at $n=10^{6}$. As a bearing material a tin-based white metal has been selected. Further data are: $p_{\mathrm{a}}=2.10^{6} \mathrm{~N} / \mathrm{m}^{2}, r=2.10^{-3} \mathrm{~m}$, and $\Delta r=4.10^{-6} \mathrm{~m}$. In a brochure on white metals, a $k$ value of approx. $1.10^{-16} \mathrm{~m}^{3} / \mathrm{Nm}$ is found for an application under conditions of boundary lubrication.

From the data on $\Delta r$ and $d$, it follows that the value of $\sigma$, as well as the maximum allowable value of $\delta$, amounts to $20.10^{-4}$. The product $p_{\mathrm{a}} k n$ has a value of $2.10^{-4}$. With $\sigma=20.10^{-4}$, Figure 4 yields a $\delta$ value of slightly below $19.10^{-4}$, which is only marginally smaller than the required maximum value of $20.10^{-4}$. In view of the fact that a $k$ value (taken from the brochure) is only a rough estimate of the value which really applies in practice, the above margin between the calculated and the required $\delta$ values should be considered to be too small. 
It can be concluded that the value of $k$ should be verified experimentally, prior to practical application of the bearing material in question.

References 1. Lancaster, J.K., 'Dry bearings: a survey of materials and factors affecting their performance', Trib. Int, 6, 219 (1973).

2. Fein, R.S., 'Boundary lubrication', in Handbook of Lubrication, Theory and Practice of Tribology. II - Theory and Design, ed. E. Richard Booser, CRC Press, 1984, pp. 49-68.

3. de Gee, A.W.J., 'Selection of materials for tribotechnical applications', Trib. Int., 11, 233 (1979).

4. Mens, J.W.M., Huis in't Veld, A.J., and de Gee, A.W.J., 'Wear of metal matrix composites (MMCs) tested under conditions of boundary lubrication', Eurotrib '93, Proc. 6th Int. Cong. on Tribology vol. 5, ed. M. Kozma, Hungarian Academy of Sciences, Budapest, 1993, p. 76.

5. Holm, R., Electrical Contacts, Almquist \& Wiksell, Stockholm, 1946.

6. Archard, J.F., 'Contact and rubbing flat surfaces', J. Appl. Phys., 24, 981 (1953).

7. Ligterink, D.J., 'Calculation of wear (f.i. wear modulus) in the plastic cup of a hip joint prosthesis', Wear, 35, 113 (1975).

8. Rymuza, Z., 'Trybologia polimeróv ślizgowych', Wydawnicta Naukowo-Techniczne Warszawa, 1986.

9. Landheer, D., Faessen, J.P.M., and de Gee, A.W.J., 'A transition diagram for plain journal bearings', Trib. Trans., 33, 418 (1990).

10. Fuller, D.D., Theory and Practice of Lubrication for Engineers, John Wiley \& Sons, Inc,, New York, 1956.

11. Bronshtein, I.N., and Semendyayev, K.A., A Guide Book to Mathematics, Springer Verlag, New York, 1973.

12. Odi-Owei, S., and Schipper, D.J., 'Tribological behaviour of unfilled and composite polyoxymethylene', Wear, 148, 363 (1991).

13. Lancaster, J.K., 'Dry rubbing bearings', in Tribology Handbook, ed. M.J. Neale, Butterworths, London, 1973.

14. Honselaar, A.C.M., and de Gee, A.W.J., 'Dynamic loadability of polymetal friction couples', in Eurotrib '89, Proc. 5th Intern. Cong. on Tribology, ed. K. Holmberg, Tribology Society, Helsinki, 1989, p. 282. 